\title{
Assessing practical laparoscopic training in certified Training Centers of the Gynecological Endoscopy Working Group (AGE) of the German Society of Gynecology and Obstetrics (DGGG)
}

\author{
Andreas Hackethal ${ }^{1}$ (D) . Franz-Erich Solomayer ${ }^{2}$. Uwe A. Ulrich ${ }^{3} \cdot$ Sara Brucker $^{4} \cdot$ Bernd Bojahr $^{5} \cdot$ Bernd Holthaus $^{6}$. \\ Stefan Rimbach ${ }^{7}$
}

Received: 17 April 2019 / Accepted: 6 August 2019 / Published online: 21 August 2019

(c) The Author(s) 2019

\begin{abstract}
Purpose This study was performed to assess the practical laparoscopic training in Gynecological Endoscopy Working Group (AGE) certified Training Centers (TC) and evaluate the possible implementation for a manual dexterity skills-training within the Minimal Invasive Surgery (MIC) certification process.

Material and methods An online questionnaire was developed and the link provided for the heads of the AGE TC. The questionnaire comprised topics on TC organization, practical training performance and perspectives for future training and demographic data.

Results Response rate was 78.9\% (15/19) of AGE TC. Grasping for the basic and suturing exercises for the advanced curricula, respectively, are thought to be of highest value (each $1.0 \pm 0$, on a scale from $1=$ very valuable to $6=$ not at all valuable). Most valuable parameter in assessing training was thought to be pressure/tension with $1.80 \pm 1.08$ The most valuable training capacity was considered for box training under supervision $(1.27 \pm 0.59)$ and feed-back box training with direct evaluation of various surgical skills $(1.40 \pm 0.63)$. Supervised box training was also thought to have the most positive influence on surgical performance $(1.33 \pm 0.49)$. The majority of respondents $(86.7 \%)$ were qualified with the highest MIC certification and additional $66.7 \%$ were sub-specialized Gynecological Oncologists.

Conclusion The AGE certified TC offer a structured curriculum with emphasis on practical training. The results of this questionnaire and the additional respondents comments on value and future perspectives/changes of practical training support the concept and the implementation of a skills-training to the AGE MIC concept.
\end{abstract}

Keywords Laparoscopy training $\cdot$ Surgical education $\cdot$ Manual skills $\cdot$ Manual dexterity training

Andreas Hackethal

1 Frauenklinik an der Elbe, Oberbaumbrïcke 1, 20457 Hamburg, Germany

2 Universitätsklinik Homburg/Saar, Kirrberger Str, 66424 Homburg, Germany

3 Martin-Luther Krankenhaus, Caspar-Theyß-Straße 27-31, 14193 Berlin, Germany

4 Universitäts-Frauenklinik, Calwerstrasse 7, 72076 Tübingen, Germany

5 Klinik für MIC, Kurstraße 11, 14129 Berlin-Zehlendorf, Germany

6 Krankenhaus St. Elisabeth gGmbH, Lindenstraße 3-7, 49401 Damme, Germany

7 Krankenhaus Agatharied GmbH, Norbert-Kerkel-Platz, 83734 Hausham, Germany

\section{Introduction}

The Gynecological Endoscopy Working Group (AGE) strives for clinical excellence with a structured training and education curriculum in gynecological hysteroscopy and laparoscopy [1]. The AGE, founded in 1993, is part of the German Society of Gynecology and Obstetrics (DGGG) and represents the largest Workgroup of the German Society for Gynecology and Obstetrics (DGGG) with over 1800 members in 2019.

To standardize training and education as well as promote theoretical and clinical expertise, a graded certification module, Minimal Invasive Surgeon (MIC), was developed by the AGE board, discussed and optimized by the AGE council and accepted by the members during a general assembly in 2005 [1, 2]. Gynecological specialists and trainees as members of the AGE can apply for accreditation. During the last 
decade, there was a continuously increasing number of certified AGE members with 1144 certified members (MIC 1: 612, MIC II: 439, MIC III: 93) and 24 Training Centers in 2019. Requirements for application and certification are a defined number of completed hysteroscopic and laparoscopic procedures, conference and work-shop attendances and visits within other departments i.e. A full list of requirements for the different MIC certificates are listed in detail on the AGE homepage (www.ag-endoskopie.de). An overview of personal requirements for MIC and institutional requirements for Trainings Center certification are summarized in Tables 1 and 2.

To further develop and add objective transparency to the MIC certification, the AGE board and members decided in 2016 to incorporate a scientifically based manual dexterity training or skills training to the already established individual certification requirements.

To better define and standardize specific box trainer or box trainer tasks and educational criteria for the skills training, this study aims to assess how the current practical laparoscopy training within certified AGE TC is performed and how to enquire on further perspectives of endoscopy training and skills training within the AGE TC.

\section{Material and methods}

An online questionnaire with 29 questions in three parts was developed. The first part comprised nine questions on Training Center organization and their course volumes. The questionnaires second part assessed practical laparoscopic training parameters. Demographic data were collected within the last six questions of part three.
Table 2 The requirements for certified AGE Training Centers are summarized

\begin{tabular}{ll}
\hline AGE Training Center parameter & Requirements \\
\hline Head & At least MIC II certified \\
Basic curriculum & Conduct at least one per year \\
Advanced curriculum & Conduct at least one per year \\
Operative Endoscopies & Perform at least 800 per year \\
Operative Laparoscopies & Hysterectomy \\
(at least out of 4 categories) & Myomectomy \\
& Lymphadenectomy \\
& Resektion of deep infiltrat- \\
& ing endometriosis \\
& Suspension surgeries \\
& Organ sparing excisions \\
& Organ sparing ovarian cys- \\
& tectomies \\
& Organ sparing ectopic preg- \\
& nancies \\
Operative Hysteroscopies & Myomectomy \\
(at least out of 3 categories) & Polyp resection \\
& Septum dissection \\
& Endometrium ablation \\
Visits & Lysis of Synechia (III-IV ${ }^{\circ}$ ) \\
\hline
\end{tabular}

A google account was created for this study and the questionnaire was based on Google Drive platform. The questionnaire is attached in "Appendix 1".

The heads of the AGE MIC Training Centers were invited by email to participate in this online survey between September and November 2016, with closing date 30th November 2016. At the end of October 2016 a reminder email was sent out. The answers were automatically saved on Google Drive and imported and analyzed with PSPP for iOS. Descriptive analysis was performed.

Table 1 Summaries requirements for AGE MIC (Minimally Invasive Surgeon) I to III certification

\begin{tabular}{|c|c|c|c|c|}
\hline & MIC I & MIC II & MIC III & MIC IIIa \\
\hline Member of the AGE & $*$ & $*$ & $*$ & $*$ \\
\hline Previous MIC certificate & & MIC I & MIC I and MIC II & MIC I and MIC II \\
\hline Certified Specialist Gynecology and Obstetrics & & $*$ & $*$ & $*$ \\
\hline Basic curriculum & $*$ & & & \\
\hline Advanced curriculum & & $*$ & & \\
\hline Skills Training & $*$ & $*$ & $*$ & $*$ \\
\hline Clinical visits & & & 10 days & 10 days \\
\hline Attending 4 certified conferences & & $*$ & $*$ & $*$ \\
\hline Number of performed laparoscopies & 30 (Typ I) & $\begin{array}{l}400 \text { (Typ II/III/IV) } \\
\text { at least } 20 \text { Typ III/ } \\
\text { IV }\end{array}$ & $\begin{array}{l}800 \text { (Typ II/III/IV) } \\
\text { at least } 80 \text { Typ III/ } \\
\operatorname{IV}\left({ }^{\circ}\right)\end{array}$ & $\begin{array}{l}400 \text { (Typ II/III/IV) } \\
\text { at least } 100 \text { Typ III/ } \\
\operatorname{IV}\left({ }^{\circ}\right)\end{array}$ \\
\hline $\begin{array}{l}\text { From these: Number of assisted and supervised } \\
\text { surgeries }\end{array}$ & & up to $25 \%$ & up to $50 \%$ & up to $50 \%$ \\
\hline Number of performed hysteroscopies & 20 (diagnostic) & 50 (operative) & 60 (operative) & 60 (operative) \\
\hline $\begin{array}{l}\text { From these: Number of assisted and supervised } \\
\text { surgeries }\end{array}$ & & & up to $50 \%$ & up to $50 \%$ \\
\hline
\end{tabular}

${ }^{*}$ Requirement, ${ }^{\circ}$ additional information available on AGE homepage 


\section{Results}

From 19 AGE certified Training Center (TC) in 2017, 15 (78.9\%) completed questionnaires were available for evaluation. The respondent AGE TC were certified since a mean $4.86 \pm 3.21$ years. These 15 centers conduct 22 basic and 21 advanced curricula per year. Additional surgery courses were conducted by $67 \%(10 / 15)$ of these centers. These courses focus on special surgical technics, and are named and performed yearly: hysterectomy $=1$, hysteroscopy $=1$, endometriosis $=1$, urogynecology $=4$, myomectomy $=1$, suturing skills $=1$, radical hysterectomy $=1$, cadaver workshop $=1$, hospitation with observation of live surgery $=2$.

Respondents see a focus on practical training compared to theoretical education as an important characteristic for the basic curricula $(57.3 \% \pm 17.9$ vs. $40.7 \% \pm 17.1)$ and the advanced curricula $(58.7 \% \pm 21.0$ vs. $42.7 \% \pm 19.8)$. For basic and advanced curricula, box training with different training models and Virtual Reality Simulators were more frequently used then box training with sensors to track instrument/target interactions (Table 3).

While grasping exercises were thought to be most valuable training tools for the basic curricula, needle movement and suture exercises were thought to be most valuable in advanced curricula (Table 4). Additionally, respondents valued suture training and techniques combined with standardization of suturing as highly appreciated tasks for the basic and the advanced curriculum (Table 4). For the Basic Curriculum suggested additional tasks were: 1. Use of $0^{\circ}$ and $30^{\circ}$ scopes and coordination $(3 \times), 2$. Suture techniques $(3 \times), 3$. Dissection techniques $(1 \times)$ and for the Advanced Curriculum: 1. Dissection with demonstration of anatomical structures $(1 \times), 2$. Simulation of surgical procedures $(2 \times)$, 3. Complication management $(\times 2), 4$. Standardized suture techniques $(4 \times)$ and 5 . Practical training with objective measurement of skills $(1 \times)$.

The respondents thought, there is no parameter clearly superior for evaluating participants during box training, however, assessed pressure/tension for the evaluation of tissue handling was thought to be most valuable (Table 4). Additionally, 6/15 respondents suggested further parameter to be of value. These comprised measuring blood loss in virtual reality environment, repetition of tasks and skills, tissue dissection and tissue handling, strategy in solving tasks, following instructions. Most important characteristics and features of box trainer are thought to comprise easiness and flexibility for set up and allowing for individual trocar positioning. The training models and tasks should be train relevant procedures in a structured way and be cost-effective and reusable (Table 4).

The most valuable training capacity $(1=$ very valuable, $6=$ not at all valuable) was considered for box training under supervision $(1.27 \pm 0.59)$ and feedback box training with direct evaluation of various surgical skills $(1.40 \pm 0.63)$ (Table 5). Supervised box training was also thought to have the best positive influence on surgical performance $(1.33 \pm 0.49)$ (Table 5).

Further considerations on value of training for the AGE courses were given from $67 \%(10 / 15)$ of participants. These comprise:

1. Stepwise training with animal lab and cadaver lab for experts.

2. Maximum of two participants per box trainer only.

3. Defined goal for practical training.

4. Standardization of practical laparoscopy training in between centers.

5. Courses should always comprise practical skills-training.

6. Complications management.

\section{Demographic data}

Respondents mean age was 47.5 years (SD 6.3), whilst $46.7 \%(7 / 15)$ were clinic directors, with the remaining being senior consultants $26.7 \%$ (4/15) and private practitioners $26.7 \%$ (4/15). Ten respondents (66.7\%) were additionally sub specialized Gynaecological Oncologists; the majority were qualified as MICIII surgeons [86.7\% (13/15)]. The total number of responsible held laparoscopic training courses differed individually between less than $20(6 / 15,40 \%)$ to more than 80 courses $(2 / 15,13.3 \%)$, whilst the remaining $7 / 1556.7 \%$ held between 20 and 80 courses.

Ten respondents addressed answers to most important changes in laparoscopic surgical education, these were

Table 3 An overview of what kind of practical laparoscopy trainer and training model is regularly used for basic and advanced curricula within the AGE Training Centers

\begin{tabular}{lcc}
\hline & Basic curricula & Advanced curricula \\
\hline Box trainer with improvised training models & $9 / 15(60 \%)$ & $9 / 15(60 \%)$ \\
Box trainer with standardized training models & $9 / 15(60 \%)$ & $11 / 15(73.3 \%)$ \\
Box trainer with commercially available standardized training models & $9 / 15(60 \%)$ & $9 / 15(60 \%)$ \\
Box trainer with sensors to track instrument/target interactions & $1 / 15(6.7 \%)$ & $1 / 15(6.7 \%)$ \\
Virtual Reality Simulator & $6 / 15(40 \%)$ & $7 / 15(46.7 \%)$ \\
\hline
\end{tabular}


Table 4 Summarizes answers on value of box trainer tasks, participants evaluation, box trainer features and task models from $(1=$ very valuable, $6=$ not at all valuable)

\begin{tabular}{|c|c|c|}
\hline Question & Parameter & $\begin{array}{l}(1=\text { very valuable/important to } \\
6=\text { not at all valuable/important })\end{array}$ \\
\hline \multirow{4}{*}{$\begin{array}{l}\text { Box trainer tasks to be valuable for training in basic } \\
\text { curriculum? }\end{array}$} & Grasping exercise & $1.00 \pm 0$ \\
\hline & Cutting exercise & $1.47 \pm 1.30$ \\
\hline & Needle movement exercise & $1.47 \pm 0.74$ \\
\hline & Suture exercise & $2.27 \pm 1.28$ \\
\hline \multirow{4}{*}{$\begin{array}{l}\text { Box trainer tasks to be valuable for training in } \\
\text { advanced curriculum? }\end{array}$} & Grasping exercise & $2.53 \pm 1.68$ \\
\hline & Cutting exercise & $2.67 \pm 1.80$ \\
\hline & Needle movement exercise & $1.13 \pm 0.35$ \\
\hline & Suture exercise & $1.00 \pm 0$ \\
\hline \multirow{4}{*}{$\begin{array}{l}\text { Parameter to be valuable for evaluation of participants } \\
\text { box training? }\end{array}$} & Time (to finish a task) & $1.93 \pm 0.96$ \\
\hline & $\begin{array}{l}\text { Instrument movement (Effectiveness in } \mathrm{cm} \text { to finish } \\
\text { a task) }\end{array}$ & $2.00 \pm 0.85$ \\
\hline & Errors (i.e dropping object) & $2.27 \pm 1.10$ \\
\hline & Pressure/tension (i.e for evaluation of tissue handling) & $1.80 \pm 1.08$ \\
\hline \multirow{8}{*}{$\begin{array}{l}\text { Which characteristics and features are important to be } \\
\text { combined with the ideal box trainer? }\end{array}$} & Easy to set up and flexible & $1.27 \pm 0.46$ \\
\hline & To have adjustable height & $2.33 \pm 1.11$ \\
\hline & Enable flexible trocar positioning & $1.87 \pm 1.41$ \\
\hline & Assess and save training data to enable learning curve & $2.27 \pm 0.70$ \\
\hline & Usable with plastic and organic models & $2.73 \pm 1.10$ \\
\hline & $\begin{array}{l}\text { Combinable device, allowing to track instrument } \\
\text { movement and coordination }\end{array}$ & $2.20 \pm 0.86$ \\
\hline & $\begin{array}{l}\text { Device allowing to assess pressure and tension at task } \\
\text { model }\end{array}$ & $2.27 \pm 1.03$ \\
\hline & Ability to choose instrument & $2.07 \pm 1.39$ \\
\hline \multirow{7}{*}{$\begin{array}{l}\text { Which features of task models for the box trainer are } \\
\text { of important value? }\end{array}$} & Easy to purchase & $1.73 \pm 0.70$ \\
\hline & Training of relevant procedures (i.e Suturing) & $1.33 \pm 0.62$ \\
\hline & Cost effective and reusable & $1.53 \pm 0.83$ \\
\hline & Ideally close to reality (i.e simulation of bleedings) & $2.20 \pm 1.32$ \\
\hline & Structured and with instructions & $1.53 \pm 0.74$ \\
\hline & Comparable to other courses & $1.80 \pm 0.94$ \\
\hline & Easy to clean & $1.60 \pm 0.74$ \\
\hline
\end{tabular}

Table 5 Summarizes estimated training capacity, ability to evaluate the participant and positive influence on surgical performance with different training models $(1=$ very valuable, $6=$ not at all valuable $)$

\begin{tabular}{llcc}
\hline Training model & Training capacity & $\begin{array}{l}\text { Ability to evaluate the } \\
\text { participant }\end{array}$ & $\begin{array}{c}\text { Positive influence on } \\
\text { surgical performance }\end{array}$ \\
\hline Box training & $2.13 \pm 0.99$ & $2.53 \pm 0.92$ & $2.13 \pm 0.83$ \\
Box training under supervision & $1.27 \pm 0.59$ & $1.87 \pm 0.74$ & $1.33 \pm 0.49$ \\
Curricula based box training (standardized tasks) & $1.67 \pm 0.72$ & $1.87 \pm 0.74$ & $1.47 \pm 0.52$ \\
Feed-back box training with direct evaluation of various surgical & $1.40 \pm 0.63$ & $1.60 \pm 0.63$ & - \\
$\quad$ skills (instrument movement, tissue handling, errors, time) & & & $1.87 \pm 0.92$ \\
Virtual Reality Simulation training with feedback & $1.93 \pm 0.88$ & $1.93 \pm 0.88$ & 1.98 \\
\hline
\end{tabular}

thought to be 1. standardization $(4 \times), 2$. more and available Box training $(3 \times)$, measuring training $(1 \times)$, inclusion of MIC training and certification into specialization training.

\section{Discussion}

The institutionally performed endoscopic training within the certified Gynecological Endoscopy Working Group (AGE) Training Centers (TC) in Germany was assessed and evaluated in this study. With fifteen responding AGE 
TC and over 55 structured endoscopic basic and advanced training courses per year, the AGE TC are dedicated to surgical training with comprehensive experience especially in the field of endoscopic surgery. Notably, two third of TC conduct additional laparoscopy courses, apart from the AGE required curricula, which are mostly disease or organ-related procedural courses (urogynecology, hysterectomy courses i.e.).

Practical training plays an important role in the AGE TC training curricula with approximately $60 \%$ of course time. The importance of practical training is supported by scientific results showing box training on models and Virtual Reality Training being able to distinguish between novices and experts and directly translate to improved theatre performance and patient outcome [3-7].

Additionally, valuable training tasks are thought to include mainly coordination training within the basic and suture training within the advanced endoscopy courses.

The AGE TC mostly use box training and virtual reality (VR) training for the practical education. They favor standardized, easy to assemble and flexible box trainer with cost effective and reusable models simulating relevant surgical procedures. The variety of examined and published box trainer tasks ranges from raw swaps for suturing to costly and complex installable pulsating organ perfusion (POP) models $[8,9]$. Furthermore, VR Trainer allow for standardized training in an abstract or near realistic environment [10]. Whereas assessment of skills during box training is mostly subjective by the supervisor or has limited validity, such as time measurement for task completion, more advanced box trainer and VR Trainer assess various parameter (i.e. distance of instrument movement, force/traction and errors).

The inclusion of VR into AGE TC and endoscopy courses is mainly a result of the AGE purchasing a VR Hysteroscopy Trainer in 2010, which can be requested by TC for educational courses. Therefore, experience with VR-trainers, the benefits of objective skills-assessment and direct feedback has gained an important role within the TC. Even though a recent meta-analysis found better laparoscopy performance and time scores in VR training compared to box-training, all other evaluated parameter, regardless of the student's level of experience or type of activity, were equivalent [11].

In our study, AGE TC favor box trainer with sensors to objectively measure instrument movement, tissue handling, errors and time for the ability to evaluate the trainee. Therefore it is likely that TC add more advanced box trainer with objective skill assessment to their curriculum. Additionally, TC estimated all examined parameters to have a valuable role in skills assessment with pressure/tension being the most valuable and errors being the least valuable parameter in this study.
Technically these advanced objective assessments can be done by sensor equipped box trainer, such as the ForceSense system (Medishield) or VR Trainer, such as the LapSim (Surgical Science) [12, 13]. However, the AGE TC clearly favor the sensor equipped box trainer over VR trainer. The similar ability to objectively assess trainees and receive instant score results after practice as well at the systems price difference may be an important reason. More than that, VR Trainer are technically more susceptible and the maintenance is more complex compared to box trainer.

The AGE graded sub-certification and the MIC certificate is unique and has become an important qualification tool within the German speaking gynecological field. Other gynecological societies as the International Society for Gynecological Endoscopy (ISGE), the Australian Gynaecological Endoscopy Society (AGES), the British Society for Gynaecological Endoscopy (BSGE) or American Association of Gynecological Laparoscopy (AAGL), to name a few, organize sub-specialized endoscopy courses with main focus on practical training as well, but without a pre-defined curriculum or skills-assessments. Only the European Society of Gynecological Endoscopy (ESGE) promotes a complex educational concept, allowing for graded accreditation [14].

The group of respondents are highly experienced and specialized practitioners with 13 out of 15 being MIC III certified and $67 \%$ being sub-specialized in gynecologic oncology. The results of this questionnaire and the additional respondents comments on value and future perspectives/changes of practical training, mainly expecting more standardization and box training with objective assessment tools, are the reason for the AGE to further develop the concept and the implementation of a skills-training to the AGE MIC concept.

\section{Compliance with ethical standards}

Conflict of interest A Hackethal declares he has no conflict of interest. F Solomayer declares he has no conflict of interest. U. Ulrich declares he has no conflict of interest. S. Brucker declares she has no conflict of interest. B. Bojahr declares he has no conflict of interest. B. Holthaus declares he has no conflict of interest. S Rimbach declares he has no conflict of interest.

Human and animal rights statement This article does not contain any studies with human participants or animals performed by any of the authors.

Informed consent Informed consent was obtained from all individual participants included in this study.

Open Access This article is distributed under the terms of the Creative Commons Attribution 4.0 International License (http://creativeco mmons.org/licenses/by/4.0/), which permits unrestricted use, distribution, and reproduction in any medium, provided you give appropriate credit to the original author(s) and the source, provide a link to the Creative Commons license, and indicate if changes were made. 


\section{Appendix 1}

Appendix 1

Exert from the online-questionnaire

\section{MIC Educational Centres}

1.1 Which AGE Teaching Centre do you belong to?

1.2 For how many years has your clinic been certified as AGE Teaching Centre?

1.3 How many MIC Basic curricula does your AGE Teaching Centre perform yearly?

1.4 How many MIC Advanced curricula does your AGE Teaching Centre perform yearly?

1.5 Do you offer additional surgical teaching curricula?

Yes/No

If Yes, which ones?

1.6 How is the average emphasis between laparoscopy and hysteroscopy training within MIC Basic courses?

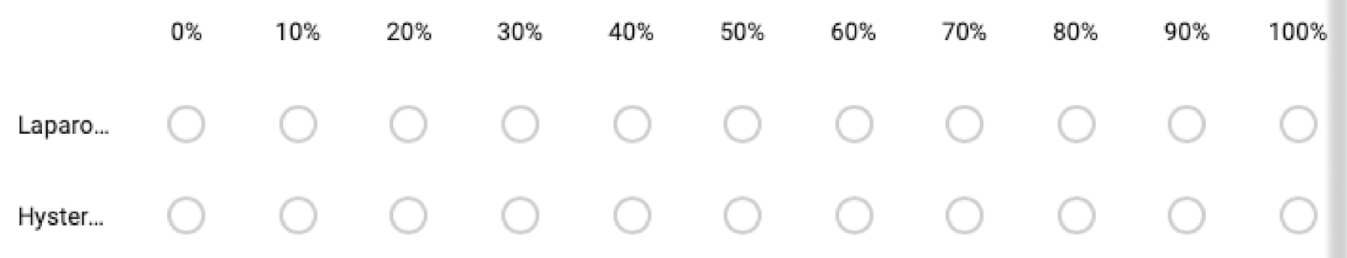

1.7 How is the average emphasis between laparoscopy and hysteroscopy training within MIC Advanced courses?

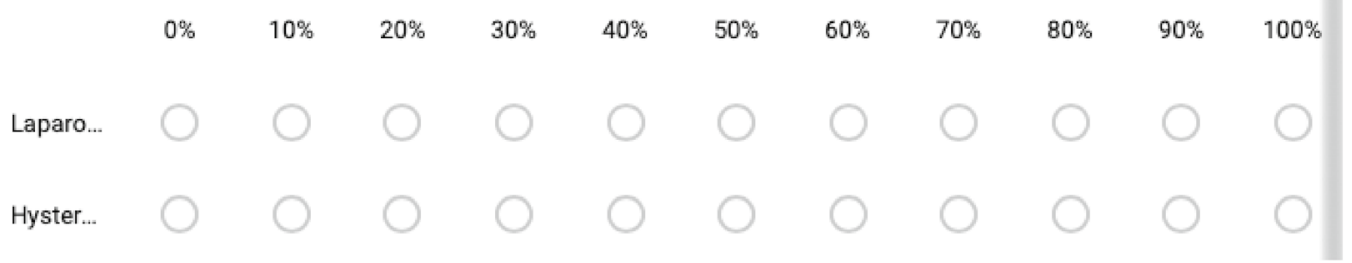

1.8 How is the average emphasis between theory and practical training within MIC Basic courses?

$\begin{array}{llllllllllll} & 0 \% & 10 \% & 20 \% & 30 \% & 40 \% & 50 \% & 60 \% & 70 \% & 80 \% & 90 \% & 100 \% \\ \text { Theorie } \quad \bigcirc & \bigcirc & \bigcirc & \bigcirc & \bigcirc & \bigcirc & \bigcirc & \bigcirc & \bigcirc & \bigcirc & \bigcirc \\ \text { Praxis } & \bigcirc & \bigcirc & \bigcirc & \bigcirc & \bigcirc & \bigcirc & \bigcirc & \bigcirc & \bigcirc & \bigcirc & \bigcirc\end{array}$


1.9 How is the average emphasis between theory and practical training within MIC Advanced courses?

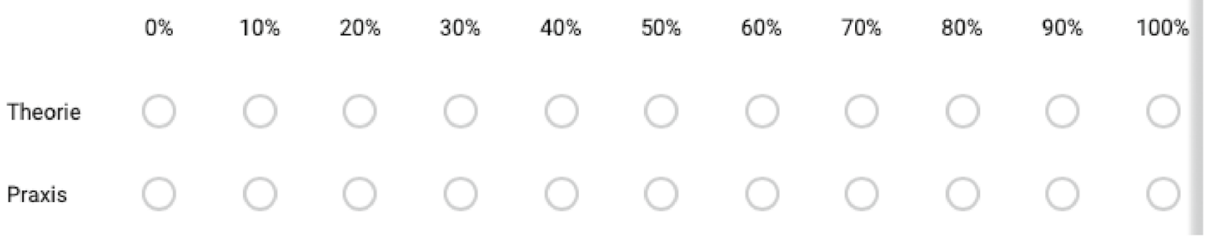

\section{Practical Training}

2.1 Which laparoscopy training models do you use for basic MIC curricula?

$\begin{array}{ll}\text { Box trainer with improvised training models } & -- \\ \text { Box trainer with standardized training models } & -- \\ \text { Box trainer with commercially available standardized training models } & -- \\ \text { Box trainer with sensors to track instrument/target interactions } & -- \\ \text { Virtual Reality Simulator } & --\end{array}$

2.2 Which box trainer tasks are important for basic MIC curricula (1 very relevant - 6 very irrelevant)

Grasping exercise
Cutting exercise
Needle movement exercise
Suture exercise

2.3 Do you think additional tasks are useful?

If Yes, which ones?
Yes/No

$--------------$

2.4 Which laparoscopy training models do you use for advanced MIC curricula?

Box trainer with improvised training models

Box trainer with standardized training models

Box trainer with commercially available standardized training models

Box trainer with sensors to track instrument/target interactions

Virtual Reality Simulator

2.5 Which box trainer tasks are important for advanced MIC curricula (1 very relevant - 6 very irrelevant)

Grasping exercise

Cutting exercise

Needle movement exercise

Suture exercise

2.6 Do you think additional tasks are useful?

Yes/No 
If Yes, which ones?

2.7 How high do you estimate the trainees benefit in practical experience with following training methods ( 1 very relevant -6 very irrelevant)

$\begin{array}{ll}\text { Box trainer with improvised training models } & -- \\ \text { Box trainer with standardized training models } & -- \\ \text { Box trainer with commercially available standardized training models } & -- \\ \text { Box trainer with sensors to track instrument/target interactions } & -- \\ \text { Virtual Reality Simulator } & --\end{array}$

2.8 How comprehensive do you estimate the possibility to assess trainees during practical training with various training models ( 1 very good -6 not good at all)

Box trainer with improvised training models

Box trainer with standardized training models

Box trainer with commercially available standardized training models

Box trainer with sensors to track instrument/target interactions

Virtual Reality Simulator

2.9 Which measurable box trainer parameter are relevant to assess manual skills? (1 very relevant -6 very irrelevant)

Time (to finish a task)

Instrument movement (Effectiveness in $\mathrm{cm}$ to finish a task)

Errors (ie dropping object)

Pressure/tension (ie for evaluation of tissue handling) - -

2.10 Do you think of additional parameter as relevant?

Yes/No

If Yes, which ones?

2.11 Which ideal characteristics and possibilities should a box trainer have? (1 very important - 6 not at all important)

Easy to set up and flexible

To have adjustable hight

Enable flexible trocar positioning

Assess and save training data to enable learning curve

Usable with plastic and organic models

Combinable device, allowing to track instrument movement and coordination

Device allowing to assess pressure and tension at task model

Ability to choose instrument

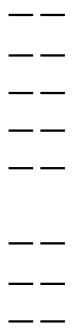

2.12 Which characteristics of training models for box trainers are important (1 very important - 6 not at all important)

Easy to purchase

Training of relevant procedures (ie Suturing)

$--$

Cost effective and reusable

Ideally close to reality (ie simulation of bleedings)

Structured and with instructions

Comparable to other courses 
Easy to clean

2.13 How high do you estimate the influence of laparoscopic training on surgical skills (1 very high -6 very low)

Box trainer with improvised training models

Box trainer with standardized training models
Box trainer with commercially available standa

$--$

Virtual Reality Simulator

2.14 Are there any additional ideas on laparoscopy training that may add value to the AGE MIC training courses?

\section{Demographic data}

3.1 How old are you?

3.2 What is your clinical position?

$$
\begin{aligned}
& \text { "Assistant doctor“" } \\
& \text { "Specialist doctor" } \\
& \text { "Consultant doctor" } \\
& \text { ",deputy director" } \\
& \text { "Clinic director" } \\
& \text { "private practitioner" }
\end{aligned}
$$

3.3 Are you sub specialized?

Gynecological Oncology
Prenatal Medicine
Endocrinology
none

3.4 How is your MIC certification grade?

MIC I

MIC II

MIC III

none

3.5 How many MIC Training courses did you conduct as trainer?

$$
\begin{aligned}
& <20 \\
& 20-40 \\
& 40-60 \\
& 60-80 \\
& >80
\end{aligned}
$$

\subsection{From your point of view, what would be the most important change within the future surgical laparoscopic training?}

\section{References}

1. Rimbach S, Ulrich U, Bojahr B, Holthaus B, Solomayer E (2015) Qualitätssicherung, Nachwuchsförderung und wissenschaftliches Engagement. Geburtshilfe Frauenheilkd 75:113-117

2. DeWilde RL, Hucke J, Kolmorgen K, Tinneberg H (2011) Recommendations by the gynecologic endoscopy working group of the german society of obstetrics and gynecology for the advancement of training and education in minimal-access surgery. Arch Gynecol Obstet 283:509-512

3. Alaker M, Wynn GR, Arulampalam T (2016) Virtual reality training in laparoscopic surgery: a systematic review and metaanalysis. Int J Surg 29:556-562

4. Janssens S, Beckmann M, Bonney D (2015) Introducing a laparoscopic simulation training and credentialing program in 
gynaecology: an observational study. Aust N Z J Obstet Gynaecol 55:374-378

5. Vanderbilt AA, Grover AC, Pastis NJ et al (2014) Randomized controlled trials: a systematic review of laparoscopic surgery and simulation-based training. Glob J Health Sci 7:310-327

6. Hackethal A, Immenroth M, Bürger T (2006) Evaluation of target scores and benchmarks for the traversal task scenario of the minimally invasive surgical trainer-virtual reality (MIST-VR) laparoscopy simulator. Surg Endosc 20:645-650

7. Cox T, Seymour N, Stefanidis D et al (2015) Moving the needle: simulation's impact on patient outcomes. Surg Clin North Am 95:827-829

8. Kalvach J, Ryska O, Ryska M (2016) Existing laparoscopic simulators and their benefit for the surgeon. Rozhl Chir 95:4-12

9. Enciso S, Díaz-Güemes I, Pérez-Medina T et al (2016) Validation of a structured intensive laparoscopic course for basic and advanced gynecologic skills training. Int J Gynaecol Obstet 133:241-244

10. Yiannakopoulou E, Nikiteas N, Perrea D, Tsigris C (2015) Virtual reality simulators and training in laparoscopic surgery. Int J Surg 13:60-64
11. Guedes HG, Ferreira ZMCC, de Sousa Leao LR et al (2019) Virtual reality simulator versus box-trainer to teach minimally invasive procedures: a meta-analysis. Int J Surg 61:60-68

12. Hardon SF, Horeman T, Bonjer HJ et al (2018) Force-based learning curve tracking in fundamental laparoscopic skills training. Sure Endosc 32:3609-3621

13. Tanoue K, Uemura M, Kenmotsu H et al (2010) Skills assessment using a virtual reality simulator, LapSim, after training to develop fundamental skills for endoscopic surgery. Minim Invasive Ther Allied Technol 19:24-29

14. Campo R, Wattiez A, Tanos V et al (2016) Gynecological endoscopic surgical education and assessment. A diploma program in gynecological endoscopic surgery. Gynecol Surg 13:133-137

Publisher's Note Springer Nature remains neutral with regard to jurisdictional claims in published maps and institutional affiliations. 